\title{
O ENVELHECIMENTO INFLUENCIAAS HABILIDADES PRAGMÁTICAS, LÉXICO-SEMÂNTICAS E PROSÓDICAS DO HEMISFÉRIO DIREITO? ${ }^{1}$
}

\author{
Rochele Paz Fonseca ${ }^{2}$ \\ Gabriela Peretti Wagner ${ }^{3}$ \\ Juciclara Rinaldi ${ }^{4}$ \\ Maria Alice de Mattos Pimenta Parente ${ }^{5}$
}

\section{Resumo}

As habilidades lingüísticas formais são relacionadas ao papel do hemisfério cerebral esquerdo, enquanto as habilidades funcionais são ligadas predominantemente à ativação do hemisfério direito. A função linguagem no envelhecimento tem sido freqüentemente estudada. No entanto, encontram-se na literatura, geralmente, pesquisas sobre os aspectos lingüísticos formais em adultos idosos, sendo os funcionais ainda pouco investigados. O objetivo do presente estudo foi averiguar se o envelhecimento influencia no desempenho comunicativo associado ao hemisfério direito. Participaram da pesquisa 40 adultos jovens e 40 adultos idosos, de ambos os sexos, com escolaridade igual ou superior a oito anos de estudo, sem relato de quaisquer patologias sensoriais, neurológicas ou psiquiátricas. Foram incluídos indivíduos com escore no Mini Exame do Estado Mental superior a 23 pontos (adultos idosos) e ausência de depressão na Escala de Depressão Yesavage. O desempenho comunicativo foi examinado através da Bateria Montreal de Avaliação da Comunicação, versão Brasileira do instrumento canadense Protocole Montréal d'Évaluation de la Communication - tarefas de interpretação de metáforas, interpretação de atos de fala indiretos, evocação lexical, julgamento semântico e compreensão e

\footnotetext{
${ }^{1}$ Agradecimento ao CNPq pelo financiamento dessa pesquisa. Esse estudo é derivado da Dissertação de Mestrado da primeira autora, sob orientação da quarta autora.

${ }^{2}$ Doutora e Mestre em Psicologia do Desenvolvimento (Universidade Federal do Rio Grande do Sul — UFRGS / Universidade de Montreal). Professora Adjunto da Faculdade de Psicologia e do Programa de Pós-Graduação em Psicologia (ênfase Cognição Humana) da Pontifícia Universidade Católica do Rio Grande do Sul, PUCRS. Coordenadora do Grupo de Pesquisa Neuropsicologia Clínica e Experimental. E-mail: rochele. fonseca@gmail.com

${ }^{3}$ Mestre e Doutoranda em Psicologia do Desenvolvimento, UFRGS. E-mail: gabrielapwagner@gmail.com

${ }^{4}$ Mestre em Psicologia do Desenvolvimento, UFRGS. E-mail: juciclara@terra.com.br

${ }^{5}$ Doutora em Psicologia pela Universidade de São Paulo (USP). Professora Adjunta da Graduação em Psicologia, Instituto de Psicologia, Departamento de Psicologia do Desenvolvimento e da Personalidade, e do Programa de Pós-Graduação em Psicologia da UFRGS. E-mail: malicemp@terra.com.br
} 
produção prosódicas. A análise estatística dos dados foi realizada com o Teste $t$ de Student. Os idosos apresentaram um desempenho significativamente inferior ao dos jovens na maioria das tarefas lingüísticas, com exceção da interpretação de atos de fala indiretos, prosódia lingüística - repetição e do julgamento semântico. A maior dificuldade de processamento comunicativo observada nos idosos não representou um deficit lingüístico, mas sim um efeito de idade no seu estilo comunicativo ou mudanças pragmáticas.

Palavras-chave: Comunicação. Linguagem. Hemisfério Direito. Envelhecimento.

\section{Introdução}

Contextualizado na área de neuropsicologia do desenvolvimento, o presente estudo teve por objetivo verificar a influência do envelhecimento no processamento comunicativo relacionado predominantemente ao papel do hemisfério cerebral direito. O enfoque nas habilidades do hemisfério direito (HD) implica o estudo da função comunicativa como um todo, abrangendo aspectos lingüísticos, paralingüísticos e extralingüísticos, ao invés de apenas se investigarem os componentes formais da linguagem verbal, de especialidade do hemisfério esquerdo (HE). Essa dicotomia entre os dois lados do cérebro está norteada pelo conceito de dominância cerebral, isto é, noção de que a função linguagem localiza-se em um dos hemisférios, considerado o dominante - o HE, sendo o HD o não-dominante (CALDAS, 1999).

As especializações hemisféricas para a função linguagem relacionam-se aos componentes lingüísticos. Os componentes fonologia (compreensão e produção dos sons da língua), morfologia (compreensão e produção dos morfemas, menores unidades com significado), sintaxe (construção de sentenças), e semântica do sentido literal (significado das unidades lingüísticas explícitas) são considerados aspectos lingüísticos estruturais, ou seja, relacionados à forma da língua. Esses componentes estão associados a uma ativação predominante de regiões cerebrais do HE (BINDER, 1997; GERNSBACHER; KASCHAK, 2003; WALTER et al., $2005)^{6}$. Em complementaridade, os componentes prosódico (compreensão

${ }^{6}$ Walter, Nathalie et al. FMRI Brain Interactivity Analysis of Phonological and Semantic Word Processing. In: HUMAN Brain Mapping (HBM). Toronto, 2005. Poster. 
e produção de entonações vocais) e pragmático (intenção comunicativa dos interlocutores) podem ser categorizados como aspectos funcionais da linguagem, isto é, inerentes ao uso da língua e à adaptação das informações lingüísticas aos diferentes contextos interacionais. Esses dois últimos componentes têm como correlatos neurais predominantes zonas do HD (HARLEY, 2001; JOANETTE; GOULET; HANNEQUIN, 1990).

Apesar de os pesquisadores do campo de estudo neuropsicológico tenderem a analisar amostras de indivíduos com lesões neurológicas, o interesse em pesquisas neuropsicológicas com populações normais vem crescendo (BRICKMAN et al., 2006), incluindo participantes idosos. No âmbito do Brasil, a expectativa de vida atual é de 69 anos, de acordo com dados da World Health Organization (WHO) (WORLD..., 2003). Em termos absolutos, estima-se que os idosos constituem cerca de $10 \%$ da população brasileira, o que corresponde a cerca de 14,5 milhões de pessoas com idade igual ou superior a 60 anos (BRASIL, 2004). Portanto, estudos avaliando esse intervalo etário serão cada vez mais relevantes, frente à necessidade de atender adequadamente à parcela idosa da população.

No que concerne ao desenvolvimento da linguagem durante o envelhecimento, Brandão e Parente (2001) comentam que tal temática tem sido um foco cada vez mais freqüente de pesquisas nas áreas da Psicologia e da Lingüística. Os principais objetivos dessas investigações resumem-se em identificar transformações e detectar os fatores etiológicos de possíveis modificações na linguagem decorrentes do envelhecimento. No entanto, infelizmente, a relação entre o processo de envelhecimento e as habilidades funcionais da linguagem ou comunicativas vem sendo investigada apenas em nível internacional, com exceção de alguns estudos nacionais sobre processamento discursivo em idosos (BRANDÃO, 2002; PARENTE; CAPUANO; NESPOLOUS, 1999). Até onde se sabe, a presente investigação apresentará de modo pioneiro resultados sobre três processamentos comunicativos avaliados em conjunto na população idosa: pragmático-inferencial, léxico-semântico e prosódico. Dessa forma, a verificação do desempenho comunicativo durante a adultez pode contribuir com o acréscimo de novos dados sobre características das funções comunicativas de grande ativação do HD no envelhecimento. 


\section{Funções Comunicativas do HD no Envelhecimento}

Na literatura consultada, são poucos os estudos sobre o efeito do envelhecimento no processamento das funções comunicativas ligadas ao HD. Meier e Thompson (1983) e Hausmann, Güntürkün e Corballis (2003), por exemplo, mencionam que as funções dependentes desse hemisfério sofrem declínio com o avançar dos anos, em populações normais, incluindo habilidades verbais em nível lexical, percepção visuo-espacial e memória. As investigações que permitiram essas conclusões não incluem, no entanto, tarefas específicas de comunicação, ou essas são abordadas com pouca expressividade em face da ênfase dada às habilidades visuo-espaciais.

O HD vem sendo relacionado a quatro processamentos comunicativos desde a década de 1970: discursivo, pragmático-inferencial, léxicosemântico e prosódico ${ }^{7}$. As habilidades discursivas geralmente são examinadas em tarefas de discurso narrativo e de discurso conversacional. No que concerne à compreensão do discurso narrativo, por exemplo, Parente, Capuano e Nespoulous (1999), ao estudarem o recontar de histórias, não encontraram diferenças significativas entre adultos jovens e idosos quanto à quantidade de inferências realizadas ${ }^{8}$. No entanto, identificaram que os jovens lembravam de uma maior quantidade de informações da narrativa. Sasser-Coen (1993) reforça tal idéia, na medida em que salienta a maior ênfase dada pelos idosos aos aspectos gerados pela experiência pessoal.

Quanto ao processamento pragmático-inferencial, as inferências são avaliadas geralmente em provas de compreensão de sentenças metafóricas, atos de fala indiretos (pedidos não-literais), provérbios, humor, moral de histórias, etc. Hamm e Hasher (1992) evidenciaram declínio no processamento inferencial na terceira idade, em tarefas nas quais os participantes deveriam julgar se as palavras apresentadas eram ou não coerentes com a interpretação esperada de uma narrativa. Os idosos efetuaram mais inferências incorretas do que os adultos jovens.

No que diz respeito ao processamento léxico-semântico, esse é associado à ativação bilateral dos hemisférios cerebrais (JOANETTE; GOULET; HANNEQUIN, 1990). É investigado através de tarefas que envolvem nomeação, associação semântica, decisão lexical, fluência verbal etc.

\footnotetext{
${ }^{7}$ Para uma revisão aprofundada ver Joanette, Goulet e Hannequin (1990) e Parente (2006).

${ }^{8}$ Inferências são representações mentais construídas pelo indivíduo na compreensão de unidades lingüísticas escritas ou orais, mediante a interação entre as informações explicitadas pelo interlocutor e o conhecimento de mundo do leitor ou ouvinte (GUTIÉRREZ-CALVO, 1999).
} 
Como ilustração, Mansur et al. (2006) não encontraram diferenças em um teste de nomeação de figuras entre adultos jovens e idosos.

Outra habilidade paralingüística também necessária para uma comunicação efetiva em diferentes contextos é a compreensão e produção de prosódia lingüística e emocional, avaliadas, respectivamente, com frases interrogativas, afirmativas e imperativas e com sentenças expressando diferentes sentimentos. Kiss e Ennis (2001), por exemplo, com o intuito de investigarem a percepção de afeto no discurso em adultos idosos e jovens, observaram que os primeiros participantes foram significativamente piores na realização dessa tarefa.

O breve referencial teórico apresentado mostra que o desempenho lingüístico provavelmente é afetado de modo dissociado no decorrer do avançar cronológico. Conforme Ska e Goulet (1989), algumas habilidades mantêm-se intactas ou melhoram com o envelhecimento, como o reconhecimento lexical, a produção oral automática e a compreensão de sentenças contextualizadas.

Frente à escassez de estudos sobre habilidades comunicativas ligadas ao HD no envelhecimento em nível nacional e às controvérsias sobre a ocorrência de declínio em tais habilidades com o avançar da idade, o presente estudo torna-se relevante. Pretende-se responder, então, à seguinte questão de pesquisa: o envelhecimento influencia no processamento comunicativo relacionado ao HD?

\section{Método}

Nesta seção são descritos aspectos metodológicos do presente estudo. Primeiramente, a amostra será caracterizada. Em um segundo momento, são explicitados os critérios de inclusão e de exclusão. Na terceira subseção, são detalhados os instrumentos utilizados na coleta de dados; e na quarta, os procedimentos.

\subsection{Participantes}

Neste estudo participaram 80 indivíduos, divididos em dois grupos etários: 1) adultos jovens $(n=40)$ e 2$)$ adultos idosos $(n=40)$. A caracterização sócio-demográfica dos dois grupos pode ser visualizada na Tabela 1. 
Tabela 1 - Caracterização Sócio-Demográfica dos Grupos de Adultos Jovens e Adultos Idosos

\begin{tabular}{l|l|l}
\hline \multicolumn{1}{c|}{ Grupos* } & \multicolumn{1}{|c}{ Adultos J ovens } & \multicolumn{1}{|c}{ Adultos I dosos } \\
\hline Faixa etária & $19-35$ & $59-78$ \\
\hline Idade M (DP) & $24,20(3,61)$ & $66,70(5,29)$ \\
\hline Escolaridade M (DP) & $15,43(2,88)$ & $14,85(5,55)$ \\
\hline Sexo (F/M) & $26 / 14$ & $31 / 09$ \\
\hline $\begin{array}{l}\text { Escore freqüência hábi- } \\
\text { tos de leitura e escrita }\end{array}$ & $16,20(4,50)$ & $11,00(3,79)$ \\
\hline Escore sócio-econômico & $34,00(9,61)$ & $27,30(5,64)$ \\
\hline
\end{tabular}

*M = média; $\mathrm{DP}=$ desvio-padrão; $\mathrm{F}=$ feminino; $\mathrm{M}=$ masculino

Fonte: tabela construída para esse estudo.

A análise dos dados da Tabela 1 mostra que os dois grupos apresentaram médias de escolaridade bastante semelhantes, não sendo observada diferença significativa entre os grupos $(\mathrm{t}=0,581, \mathrm{p}=0,563)^{9}$. Nota-se que tanto no grupo de jovens como no de idosos houve uma proporção maior de participantes do sexo feminino, sem diferença significativa entre os grupos quanto a sua distribuição pelo gênero $\left(\mathrm{x}^{2}=1,526, \mathrm{p}=0,217\right)^{10}$. $\mathrm{O}$ escore médio da freqüência dos hábitos de leitura e escrita foi significativamente diferente entre os grupos: os adultos idosos apresentaram escore menor do que o dos adultos jovens $(t=5,581, p \leq 0,001)$. No que concerne ao escore sócio-econômico, os adultos jovens apresentaram um escore significativamente maior que o dos idosos $(t=3,803, p \leq 0,001)$, sendo que ambos pertencem predominantemente à classe social $\mathrm{A}$ (Critério de Classificação Econômica Brasil - CCEB).

A amostra foi selecionada na população de Porto Alegre e região metropolitana. Os adultos jovens foram recrutados em ambientes universitários e empresariais. Os indivíduos idosos foram selecionados por indicação dos adultos jovens ou em grupos de convivência de terceira idade, tais como clubes, academias, paróquias e programas universitários. A amostragem foi por conveniência.

\footnotetext{
${ }^{9}$ A comparação entre grupos quanto às variáveis escolaridade (em anos), escore médio de hábitos de leitura e escrita e escore sócio-econômico foi analisada a partir do Teste t de Student para amostras independentes.

${ }^{10}$ Para a análise comparativa entre os grupos quanto à sua distribuição por gênero, foi utilizado o Teste Quiquadrado.
} 


\subsection{Critérios de Inclusão e Exclusão}

Os critérios de inclusão foram escolaridade igual ou superior a oito anos completos de estudo e fluência no Português Brasileiro, com procedência brasileira superior a 20 anos. Além disso, só foram incluídos participantes sem quaisquer distúrbios neurológicos, psiquiátricos, sensoriais - visuais e/ou auditivos - e lingüísticos auto-relatados. Quanto aos distúrbios psiquiátricos, não foram incluídos indivíduos com depressão examinada pela Escala de Depressão Yesavage (YESAVAGE, 1986; PARENTE, 1990) ${ }^{11}$. Além disso, também não participaram desse estudo adultos idosos com escore no Mini-Mental (FOLSTEIN; FOLSTEIN; MCHUGH, 1975) ${ }^{12}$ abaixo de 24, indicativo de demência. Por fim, não foram incluídos participantes que fizessem uso de drogas psicotrópicas ou com histórico prévio ou atual de uso abusivo de álcool. Todos os critérios de inclusão e exclusão foram verificados a partir da aplicação de um questionário estruturado de dados socioculturais e aspectos da saúde.

\subsection{Instrumentos}

Nesta subseção, serão descritos os instrumentos utilizados para avaliar as habilidades comunicativas de adultos jovens e de adultos idosos. Serão abordados os seguintes aspectos: objetivo do instrumento e normas de aplicação e de pontuação.

\subsubsection{Questionário de Dados Socioculturais e de Aspectos da Saúde}

Investiga questões referentes à renda, aos hábitos culturais e comunicativos e aos antecedentes médicos (aspectos de saúde geral). O escore de freqüência de hábitos de leitura e escrita foi calculado pela soma dos pontos: quando o participante apresentava hábito de leitura ou de escrita todos os dias, recebia um escore 4, alguns dias por semana, 3 , uma vez por semana, 2, raramente, 1 e nunca, 0 . O nível sócio-econômico foi avaliado pelo Critério de Classificação Econômica Brasil (CCEB), a partir do Levantamento Sócio-Econômico/2000 do Instituto Brasileiro de Opinião Pública e Estatística (IBOPE) (ASSOCIAÇÃO..., 2003).

${ }^{11}$ PARENTE, Maria Alice de Mattos Pimenta. Versão Brasileira da Escala de Depressão Geriátrica: instrumento de avaliação. Porto Alegre: Universidade Federal do Rio Grande do Sul, 1990. Não publicada.

12 Versão adaptada para a população brasileira local por Chaves e Izquierdo (1992). 


\subsubsection{Tarefas de Avaliação dos Processamentos Pragmático-Inferen- cial, Léxico-Semântico e Prosódico da Bateria Montreal de Avaliação da Comunicação (Bateria MAC)}

Algumas tarefas da Bateria Montreal de Avaliação da Comunicação - Bateria MAC (FONSECA et al., 2007a) foram utilizadas para a investigação das habilidades de comunicação dos adultos jovens e idosos. Esse instrumento corresponde à versão Brasileira do Protocole Montréal D'Évaluation de la Communication - Protocole MEC (JOANETTE; SKA; CÔTÉ, 2004). O processo detalhado de adaptação da Bateria MAC encontra-se em Fonseca et al. (2007b). As tarefas selecionadas encontramse descritas a seguir, na ordem em que foram aplicadas. Cada item é pontuado com 0,1 ou 2, exceto nas tarefas de prosódia e julgamento semântico, em que as respostas recebem 0 ou 1 ponto:

a) interpretação de metáforas: esse subteste avalia a habilidade de compreender e explicar o sentido não-literal de sentenças. É formado por 20 frases metafóricas, sendo as dez primeiras metáforas novas, ou seja, não convencionais ou comumente utilizadas no Português Brasileiro (por exemplo, Meu pai é um pavão). As dez últimas são expressões idiomáticas (por exemplo, A mãe pisa em ovos com seus filhos). O participante é orientado a explicar o que significa a frase com suas próprias palavras;

b) evocação lexical livre: esse subteste examina a habilidade de explorar livremente a memória léxico-semântica durante a evocação de palavras. O examinador pede ao participante para dizer o maior número possível de palavras em dois minutos e 30 segundos;

c) prosódia lingüística compreensão: essa prova avalia a habilidade de percepção e identificação de padrões de entonação lingüística. É constituída de quatro sentenças com estrutura gramatical simples (sujeito-verbo-objeto) com conteúdo neutro (por exemplo, Maria vai trabalhar). Cada sentença foi previamente registrada em equipamento de áudio com três diferentes entonações lingüísticas (afirmativa, interrogativa e imperativa) para um total de doze frases, em ordem aleatória. O participante é solicitado a identificar a entonação respondendo verbalmente ou apontando uma de três opções de imagens visuais correspondentes;

d) prosódia lingüística repetição: esse subteste investiga a habilidade de 
reprodução verbal de entonações lingüísticas. Baseia-se nos mesmos quatro estímulos frasais da tarefa anterior. O participante deve repetir cada sentença com a mesma entonação identificada;

e) evocação lexical com critério ortográfico: essa tarefa avalia a exploração da memória léxico-semântica durante a evocação de palavras a partir de um critério ortográfico. O participante é orientado a dizer o maior número possível de palavras que começam com a letra $P$ em dois minutos;

f) prosódia emocional compreensão: essa tarefa investiga a percepção e a identificação de padrões de entonação emocional. É formada por doze sentenças de estrutura gramatical simples (sujeito-verbo-objeto) com um conteúdo neutro (por exemplo, Clara bate na porta). Cada sentença foi previamente registrada em equipamento de áudio com três diferentes entonações emocionais (alegria, tristeza e raiva), quatro frases para cada sentimento, apresentadas em ordem aleatória. $\mathrm{O}$ indivíduo avaliado é solicitado a identificar a entonação através de uma resposta verbal ou apontando para uma das três imagens apresentadas - face alegre, triste ou colérica;

g) prosódia emocional repetição: esse subteste averigua a habilidade de reprodução verbal de entonações emocionais, com base nas mesmas quatro sentenças da tarefa anterior. $\mathrm{O}$ examinador orienta $\mathrm{o}$ indivíduo avaliado a repetir cada sentença com a mesma entonação identificada.

h) interpretação de atos de fala indiretos: essa tarefa examina a habilidade de compreender atos de fala diretos e indiretos a partir de um determinado contexto comunicativo. É composta por 20 situações breves distribuídas aleatoriamente; dessas, dez terminam com um ato de fala direto, em que o interlocutor quer dizer literalmente o que é dito (por exemplo, Esta nova televisão funciona muito bem, significando Esta nova televisão é boa); as outras dez são situações finalizadas com um ato de fala indireto, em que a intenção do interlocutor não está explicitada, devendo ser inferida do contexto (por exemplo, João, a porta do seu quarto está aberta significando João, fecha a porta). $\mathrm{O}$ participante deve explicar com suas próprias palavras o que a pessoa quis dizer;

i) evocação lexical com critério semântico: essa tarefa examina a memória léxico-semântica durante a evocação de palavras a partir 
de um critério semântico. O examinador pede ao examinando que diga o maior número possível de palavras que sejam roupas em dois minutos;

j) prosódia emocional produção: esse subteste investiga a habilidade de produção oral de padrões de entonação emocional, a partir de pistas contextuais. Constitui-se por três sentenças de estrutura gramatical simples que devem ser emitidas com três padrões diferentes de entonação emocional: alegria, tristeza e raiva (por exemplo, Acabei de vir do médico). O examinador lê a história e o participante deve dizer a sentença apresentada verbal e visualmente com a entonação que expressa a emoção induzida pela situação;

k) julgamento semântico: essa prova examina a habilidade de identificação de relações semânticas entre palavras, assim como de explicitá-las precisa e claramente. Os estímulos consistem em 24 pares de palavras, doze compostos por vocábulos com relação categorial (mesma categoria semântica; por exemplo, pia-tanque). Cada par de palavras é apresentado verbal e visualmente. O participante deve primeiramente dizer se há ou não relação entre as duas palavras (escore identificação); posteriormente, deve explicar qual é a relação, quando existente (escore explicação).

\subsection{Procedimentos}

Quanto aos aspectos éticos, a participação dos indivíduos no estudo foi voluntária, tendo todos assinado um Termo de Consentimento Livre e Esclarecido. O projeto desta pesquisa foi aprovado pelo Comitê de Ética em Pesquisa da Universidade Federal do Rio Grande do Sul ${ }^{13}$.

Os participantes foram testados individualmente, em um ambiente silencioso, iluminado e ventilado. Os instrumentos foram aplicados por um neuropsicólogo, em uma única sessão, com duração aproximada de uma hora e vinte minutos. As respostas dadas às tarefas da Bateria MAC foram analisadas por três juízes especialistas independentes, com base no Manual de Aplicação e Pontuação da Bateria MAC (FONSECA et al., 2007a). Um coeficiente de concordância foi estabelecido para cada item de cada tarefa, de acordo com o método e critério de Fagundes (1985). Aqueles itens com índices inferiores a 0,70 foram novamente analisados pelos três juízes

\footnotetext{
${ }^{13}$ Parecer número 2003207.
} 
em conjunto e um consenso foi estabelecido. Caso dois juízes houvessem concordado, a pontuação oriunda deste acordo era mantida como final.

Em relação aos procedimentos estatísticos, os dados foram analisados descritiva e inferencialmente. Os escores médios em cada tarefa da Bateria MAC obtidos pelos grupos foram comparados com o teste paramétrico $t$ de Student para amostras independentes. Considerou-se um nível de significância $\leq 0,05$. Utilizou-se o pacote estatístico SPSS, versão 12 .

\section{Resultados}

Na Tabela 2, são expostos os resultados da análise comparativa do desempenho nas habilidades comunicativas testadas pela Bateria MAC entre o grupo de adultos jovens e o grupo de adultos idosos.

Tabela 2 - Escores Médios e Níveis de Significância de Adultos Jovens e Adultos Idosos nas Tarefas Pragmático-Inferenciais, Léxico-Semânticas e Prosódicas.

\begin{tabular}{|c|c|c|c|}
\hline Tarefas & $\begin{array}{l}\text { Adultos jovens } \\
\text { M (DP) }\end{array}$ & $\begin{array}{l}\text { Adultos idosos } \\
\text { M (DP) }\end{array}$ & $\begin{array}{l}\mathbf{t} ; \\
\mathbf{p}^{*}\end{array}$ \\
\hline \multicolumn{4}{|l|}{ Pragmático-I inferencials } \\
\hline $\begin{array}{l}\text { I nterpretação de me- } \\
\text { táforas novas** }\end{array}$ & $16,08(2,49)$ & $14,18(2,49)$ & $\begin{array}{l}3,409 ; \\
p \leq 0,001\end{array}$ \\
\hline $\begin{array}{l}\text { I nterpretação de ex- } \\
\text { pressões idiomáticas }\end{array}$ & $17,35(1,97)$ & $15,00(2,67)$ & $\begin{array}{l}4,481 ; \\
p \leq 0,001\end{array}$ \\
\hline $\begin{array}{l}\text { Interpretação de atos } \\
\text { de fala diretos }\end{array}$ & $17,13(2,15)$ & $16,27(2,69)$ & $\begin{array}{l}1,561 ; \\
p=0,122\end{array}$ \\
\hline $\begin{array}{l}\text { Interpretação de atos } \\
\text { de fala indiretos }\end{array}$ & $15,70(2,49)$ & $15,18(1,81)$ & $\begin{array}{l}1,078 \\
p=0,285\end{array}$ \\
\hline \multicolumn{4}{|l|}{ LÉXI CO-SEMÂNTICAS } \\
\hline Evocação lexical livre & $62,18(23,68)$ & $41,73(15,82)$ & $\begin{array}{l}4,541 ; \\
p \leq 0,001\end{array}$ \\
\hline $\begin{array}{l}\text { Evocação lexical com } \\
\text { critério ortográfico }\end{array}$ & $26,78(7,08)$ & $20,63(9,12)$ & $\begin{array}{l}3,368 \\
p \leq 0,001\end{array}$ \\
\hline $\begin{array}{l}\text { Evocação lexical com } \\
\text { critério semântico }\end{array}$ & $31,35(6,57)$ & $22,93(5,55)$ & $\begin{array}{l}6,194 \\
p \leq 0,001\end{array}$ \\
\hline $\begin{array}{l}\text { J ulgamento semânti- } \\
\text { co: identificação }\end{array}$ & $23,68(0,66)$ & $23,58(0,68)$ & $\begin{array}{l}0,672 ; \\
p=0,504\end{array}$ \\
\hline $\begin{array}{l}\text { J ulgamento semânti- } \\
\text { co: explicação }\end{array}$ & $9,68(1,37)$ & $9,43(1,39)$ & $\begin{array}{l}0,810 \\
p=0,420\end{array}$ \\
\hline
\end{tabular}




\begin{tabular}{l|l|l|l}
\hline ProsódıcAs \\
\hline $\begin{array}{l}\text { Prosódia lingüística } \\
\text { compreensão }\end{array}$ & $11,70(0,69)$ & $11,00(1,62)$ & $\begin{array}{l}2,520 ; \\
\mathrm{p} \leq 0,05\end{array}$ \\
\hline $\begin{array}{l}\text { Prosódia lingüística } \\
\text { repetição }\end{array}$ & $11,83(0,50)$ & $11,40(1,32)$ & $\begin{array}{l}1,908 ; \\
\mathrm{p}=0,062\end{array}$ \\
\hline $\begin{array}{l}\text { Prosódia emocional } \\
\text { compreensão }\end{array}$ & $11,65(1,19)$ & $10,55(1,81)$ & $\begin{array}{l}3,212 ; \\
\mathrm{p} \leq 0,01\end{array}$ \\
\hline $\begin{array}{l}\text { Prosódia emocional } \\
\text { repetição }\end{array}$ & $10,07(2,13)$ & $7,78(2,79)$ & $\begin{array}{l}4,147 ; \\
\mathrm{p} \leq 0,001\end{array}$ \\
\hline $\begin{array}{l}\text { Prosódia emocional } \\
\text { produção }\end{array}$ & $14,65(3,17)$ & $12,20(4,21)$ & $\begin{array}{l}2,937 ; \\
\mathrm{p} \leq 0,01\end{array}$ \\
\hline
\end{tabular}

*M = média; $\mathrm{DP}=$ desvio-padrão; $\mathrm{t}=$ valor do Teste $\mathrm{t}$ de Student; $\mathrm{p}$ = nível de significância **As tarefas grifadas em negrito são aquelas com diferença estatisticamente significativas. Fonte: tabela construída para esse estudo.

Quanto ao processamento pragmático-inferencial, a análise comparativa na tarefa Interpretação de metáforas foi efetuada tanto para as metáforas novas quanto para as expressões idiomáticas. Do mesmo modo, na Interpretação de atos de fala indiretos, são apresentados dados para os atos diretos e os indiretos. A partir da análise da Tabela 2, observa-se que não houve diferença entre os adultos jovens e idosos na Interpretação de atos de fala indiretos, mas apenas na Interpretação de metáforas, em que os escores médios do último grupo foram inferiores aos do primeiro. No que diz respeito ao processamento léxico-semântico, constata-se que os adultos jovens emitiram, em média, mais palavras do que os idosos nas três modalidades de evocação lexical. No entanto, não houve diferenças significativas nos escores de identificação e explicação da tarefa Julgamento semântico. Por fim, nas tarefas que avaliam o processamento prosódico, os adultos idosos, em geral, apresentaram escores inferiores aos dos adultos jovens, diferença significativa em todas as tarefas prosódicas, exceto na Prosódia lingüística repetição.

\section{Discussão}

No presente estudo, objetivou-se verificar se o envelhecimento influencia no processamento comunicativo relacionado ao HD, considerando-se, particularmente, três componentes: pragmático-inferencial, léxico-semântico e prosódico. No que concerne ao processamento pragmático- 
inferencial, a comparação do escore obtido na compreensão das primeiras dez metáforas - novas — e das últimas dez metáforas - expressões idiomáticas - evidenciou que os jovens desempenharam significativamente melhor do que os idosos, ao explicarem o significado das duas modalidades de metáforas: não-familiares e familiares. Esse achado foi corroborado por Monetta, Ouellet-Plamondon e Joanette (2006), não tendo sido verificado por Champagne, Desautels e Joanette (2003).

Em um primeiro instante, poder-se-ia pensar que o processamento de inferências em geral prejudicado com o avançar da idade (MAXIM; BRYAN, 1994; MCGINNIS; ZELINSKI, 2003; RADANOVIC; MANSUR, 2002; WRIGHT; NEWHOFF, 2002), mencionado como uma habilidade de base para a compreensão metafórica (JOANETTE; GOULET; HANNEQUIN, 1990), justificaria o desempenho pior dos idosos nessa tarefa. Entretanto, a maioria dos estudos que avalia o processamento de inferências na idade adulta tem em seus métodos tarefas de compreensão textual e não de sentenças isoladas. A inferência exigida para o entendimento de uma narrativa está baseada predominantemente na associação de informações contextuais com o conhecimento prévio do indivíduo (GUTIÉRREZ-CALVO, 1999). Em contrapartida, na compreensão de metáforas, o processamento de inferências parece depender mais do conhecimento geral e lingüístico da própria pessoa do que das informações contextuais, já que essa habilidade é testada através de sentenças isoladas (BOTTINI et al., 1994; CHAMPAGNE; DESAUTELS; JOANETTE, 2003; WINNER; GARDNER, 1977), não inseridas em contextos maiores como uma narrativa. Desse modo, uma particularidade metodológica inerente a essa prova pode ter contribuído para a performance inferior dos idosos: ao ser formada por sentenças isoladas, a tarefa Interpretação de metáforas não fornece informações contextuais.

Embora a realização de inferências ocorra quando se ultrapassa o significado literal de um texto (HARLEY, 2001), e a compreensão de uma metáfora envolva operações cognitivas de percepção não-literal da similaridade entre dois termos (WINNER; GARDNER, 1977), um provável prejuízo no processamento inferencial não parece explicar o pior desempenho dos adultos idosos na presente pesquisa. Os comentários da maioria dos idosos demonstraram que inferências corretas estavam sendo efetuadas, à medida que comparavam os dois termos utilizados para formar as metáforas novas com base em suas características concretas. Assim, por 
exemplo, um dos participantes, ao explicar a metáfora "O professor é um sonífero" verbalizou "sonífero é aquele remédio que dá sono, que faz a gente dormir, ficar calmo; se o professor é comparado a um sonífero, ele deve dar sono nos alunos, deve dar uma aula monótona". Se as inferências foram processadas de modo satisfatório, outro processo cognitivo deve estar sendo utilizado com menor êxito pelos idosos do que pelos jovens. Provavelmente, eles realizaram com menos êxito a passagem do nível concreto para o abstrato.

Outro fator que pode ter, ainda, contribuído para a diferença de desempenho entre adultos jovens e idosos é a tendência dos últimos de explicarem tanto as metáforas não-familiares quanto as familiares através de comparações com experiências pessoais. Desse modo, suas explicações podem ter se tornado menos precisas. Entretanto, deve-se ressaltar que, de um modo geral, tanto jovens quanto idosos apresentaram um bom desempenho nessa tarefa, com o primeiro grupo obtendo escores correspondentes a $80,4 \%$ e $86,75 \%$ e o segundo, a $70,9 \%$ e $75 \%$ da pontuação máxima na compreensão de metáforas novas e de expressões idiomáticas, respectivamente.

No que concerne à Interpretação de atos de fala indiretos, diferentemente dos resultados da tarefa de compreensão de metáforas, o desempenho de jovens e idosos não foi significativamente distinto nas duas modalidades testadas - atos diretos ou literais e atos indiretos ou não-literais. Esse achado concorda com os resultados de Champagne, Desautels e Joanette (2003). Nesse estudo, tanto jovens quanto idosos processaram atos de fala indiretos adequadamente.

O desempenho dos idosos foi tão bem sucedido quanto o dos jovens provavelmente pela associação de dois fatores. O primeiro fator diz respeito à manutenção de um adequado processamento inferencial. A habilidade de captar a intenção do interlocutor é exigida na compreensão de atos de fala (HARLEY, 2001; JOANETE; GOULET; HANNEQUIN, 1990; SEARLE, 1969). Tal competência pragmática demanda o processamento de inferências, já que o indivíduo precisa compreender o desejo implícito do falante e fazer pressuposições com base no entendimento não-literal das informações contextuais. Na medida em que adultos jovens e idosos compreenderam os atos de fala de modo semelhante, com mais de $75 \%$ da pontuação máxima, constata-se que provavelmente não houve efeito da idade no processamento inferencial e na competência pragmática 
implicadas na realização da tarefa em discussão. O indício de preservação do delineamento de inferências corretas contraria os achados de Wright e Newhoff (2002) e de Hamm e Hasher (1992), mas está de acordo com os resultados de McGinnis e Zelinski (2003).

O segundo fator consiste na facilitação promovida pelas pistas contextuais fornecidas nas situações de atos de fala. Graças à presença de informações implícitas, mesmo em tarefas lingüísticas complexas como a de compreensão de atos de fala indiretos, as inferências são processadas com maior êxito. McGinnis e Zelinski (2003) constataram que a eficiência dessa habilidade é influenciada pela extensão dos estímulos utilizados nos diferentes instrumentos. Os autores observaram que parágrafos mais curtos podem ser menos sensíveis ao efeito da idade do que textos mais longos. Desse modo, na atual investigação, como as situações de atos de fala foram escritas em pequenas histórias, cujos textos são constituídos por, no máximo, três sentenças, talvez alguma dificuldade mais sutil na compreensão da linguagem não-literal, se existente, não tenha sido evidenciada. Além disso, essa menor extensão textual pode não ter causado a freqüente sobrecarga de memória observada em discursos maiores que acarretaria prejuízos na compreensão lingüística (LIGHT; ALBERTSON, 1988).

Em suma, não houve efeito do envelhecimento na habilidade de processamento inferencial na amostra investigada. A observação de diferenças significativas entre o desempenho de jovens e idosos na tarefa de compreensão de metáforas representa uma provável dificuldade dos adultos mais velhos em transpor com precisão conceitos apresentados no nível concreto para o nível abstrato, assim como por um estilo cognitivo menos objetivo dos participantes idosos. Além disso, a ausência de distinção entre os grupos contrastantes na tarefa de atos de fala indiretos sugere diferenças metodológicas. A maior extensão dos estímulos da prova de atos de fala representa uma maior quantidade de informações contextuais implícitas que, apesar da complexidade dessa tarefa, facilitou o processamento inferencial dos idosos.

Quanto ao processamento léxico-semântico, nas três modalidades da prova de evocação lexical, o escore total médio dos idosos foi significativamente inferior ao dos adultos jovens. Esse subteste, também conhecido como fluência verbal, foi utilizado por inúmeros pesquisadores. Os resultados sensíveis à variável idade encontrados nesse estudo estão em acordo, de um modo geral, sem considerar separadamente as modalidades, 
com os achados de Beausoleil et al. (2003) e Radanovic e Mansur (2002), embora estejam em desacordo com Brandão (2002), em cuja investigação não foram evidenciadas diferenças significativas entre jovens e idosos.

Para que hipóteses possam ser formuladas acerca das prováveis causas que levaram os idosos desta amostra a apresentarem um desempenho pior do que o dos adultos jovens, deve-se pensar nas habilidades cognitivas avaliadas na tarefa de evocação lexical ou fluência verbal. De acordo com Hughes e Bryan (2002), não há um consenso quanto a uma exclusiva função examinada por esse teste, o que é corroborado por Casanova (1987), que comenta que um teste ou subteste raramente avalia de modo específico apenas uma função mental independente. Na literatura, o declínio com o avançar da idade em duas habilidades cognitivas potencialmente subjacentes à execução da tarefa de evocação lexical é associado a um desempenho prejudicado dos idosos nesse teste: deficit de inibição (BRYAN; LUSZCZ, 2000) ou das funções executivas em geral (RODRIGUEZ-ARANDA; SUNDET, 2006) e diminuição da velocidade processual (SALTHOUSE, 1996). Em contrapartida, a manutenção ou ampliação de habilidades verbais representadas principalmente pelo conhecimento léxico-semântico é considerada como um fator que compensa o declínio nas demais habilidades cognitivas promovendo um bom desempenho nas provas de fluência verbal (GHISLETTA; LINDENBERGER, 2003; HUGHES; BRYAN, 2002; BEAUSOLEIL et al., 2003). Essas hipóteses formuladas com base na literatura não foram confirmadas por observações específicas na presente investigação, na medida em que alterações de funções executivas também teriam levado a um deficit inferencial, não encontrado na presente amostra. Além disso, uma menor velocidade de processamento teria afetado o desempenho em todas as tarefas desse estudo, o que também não ocorreu.

Formula-se a hipótese, então, de que um provável declínio nas habilidades verbais de recuperação da memória semântica pode ter contribuído para a diferença encontrada entre jovens e idosos. Um aumento da ansiedade também pode ter afetado a produtividade dos idosos, na medida em que as tarefas de evocação lexical foram as únicas com limitação de tempo.

Assim sendo, constata-se que os participantes do grupo dos idosos apresentaram maior dificuldade na evocação de vocábulos com e sem restrições específicas. O desempenho do grupo mais velho, no entanto, não 
pode ser considerado ruim. A análise de outros parâmetros quantitativos, tal como o número de categorias semânticas produzidas, e de aspectos qualitativos, como tipos de erros, tipos de estratégias e grau de prototipicalidade (representatividade de uma dada categoria semântica), poderia aprofundar o entendimento da diferença da quantidade de palavras emitidas entre os grupos de adultos jovens e idosos.

Ao contrário dos achados da tarefa de evocação lexical, no julgamento semântico não foi verificada uma diferença estatisticamente significativa entre adultos jovens e adultos idosos. O desempenho dos dois grupos foi semelhante tanto na avaliação da habilidade de identificar a presença ou a ausência de relação semântica entre dois vocábulos, como no exame da habilidade de explicar qual era essa relação. O excelente desempenho dos dois grupos contrastantes, representado por um escore médio de mais $98 \%$ da pontuação máxima, está de acordo com os achados de Taylor e Burke (2002). Esses autores constataram que as funções semânticas ao nível da palavra mantêm-se preservadas durante a fase adulta, enquanto as habilidades de recuperação fonológica e ortográfica sofrem um declínio importante. Além disso, Verhaeghen (2003), ao avaliar escores verbais em 210 investigações publicadas, evidenciou que os idosos identificam significados de vocábulos com a mesma ou com maior eficiência do que os adultos jovens devido, provavelmente, ao seu conhecimento semântico e a sua experiência verbal adquirida.

Além de uma adequada habilidade em tomar decisões quanto à existência de uma associação semântica entre duas palavras, os idosos também demonstraram fornecer explicações completas e precisas acerca de seus significados. Uma boa habilidade de interpretação do significado de palavras tão bem sucedida quando a dos jovens também foi encontrada em outros estudos (BURKE; HARROLD, 1988). É possível pensar que a ausência de diferença entre adultos jovens e idosos deveu-se à representação semântica da primeira palavra ouvida pelos participantes, que ocasionava uma ativação automática do significado dela e uma transmissão da excitação para representações de outras palavras a ela relacionadas semanticamente - efeito priming, tão eficiente na terceira idade quanto na adultez inicial (BURKE; MACKAY; JAMES, 2000). Dessa forma, ao ouvir o vocábulo maçã, o significado das demais frutas, palavras do mesmo campo semântico, era ativado, possibilitando aos idosos identificarem tão adequadamente quanto os jovens a interpretação associada do par de 
palavras maçã-ameixa.

Por fim, considera-se que o processamento léxico-semântico, avaliado pelas duas últimas tarefas discutidas, parece estar sendo realizado com eficiência pelos idosos do presente estudo. Apesar de esses participantes terem apresentado um desempenho inferior ao dos jovens na prova de evocação lexical, o efeito de idade observado não pode ser considerado um prejuízo lingüístico, tendo-se em vista que o fator ansiedade provavelmente intensificou a anomia cada vez mais freqüente com o avançar da idade. O excelente desempenho dos idosos no julgamento semântico, semelhante ao dos jovens, sugere uma adequada aplicação do conhecimento verbal e um adequado efeito priming.

No que diz respeito ao processamento prosódico, na prova de prosódia lingüística, a análise comparativa entre os grupos etários evidenciou resultados distintos nas duas modalidades avaliadas. Na compreensão desse tipo de prosódia, o desempenho dos participantes idosos foi significativamente inferior ao dos adultos jovens, enquanto que na tarefa de repetição de prosódia lingüística não houve diferença estatisticamente significativa. Embora haja inúmeros estudos sobre a influência do envelhecimento no processamento lingüístico ${ }^{14}$, na literatura específica consultada foi encontrada apenas uma investigação sobre os aspectos paralinguísticos na terceira idade. Diferentemente dos resultados encontrados no presente estudo, Wingfield, Lindfield e Goodglass (2000) não evidenciaram um desempenho inferior dos idosos na compreensão de variações de prosódia lingüística.

Uma vez que do total máximo de doze pontos ou acertos, adultos jovens e idosos obtiveram onze ou mais pontos, ou seja, compreenderam e repetiram de modo adequado mais de $90 \%$ dos estímulos das duas tarefas, considera-se que o último grupo apresenta habilidades preservadas de processamento prosódico. Então, de uma forma geral, pode-se afirmar que, mesmo na ausência de normas de desempenho, os participantes dos dois grupos apresentaram habilidades eficientes de compreender e de repetir sentenças com variações entonacionais de prosódia lingüística.

Quanto à tarefa de prosódia emocional, identificou-se uma diferença significativa em favor dos jovens nas três modalidades. Os adultos jovens compreenderam, repetiram e produziram, com maior êxito do que os adultos maduros, sentenças com variações entonacionais indicativas de

${ }^{14}$ Para uma revisão, ver Maxim e Bryan (1994). 
tristeza, alegria ou raiva. Assim como para o processamento de prosódia lingüística, encontra-se na literatura uma lacuna de investigações específicas sobre os efeitos da idade na compreensão e na produção de prosódia emocional. As pesquisas com populações idosas neurologicamente saudáveis investigam, em geral, apenas as habilidades de compreensão desse tipo de prosódia. Dessa forma, os resultados encontrados no presente estudo concordam com os achados de Kiss e Ennis (2001), que constataram que idosos compreendem menos do que jovens diferentes estados afetivos através de variações entonacionais. Vão, também, ao encontro do efeito de idade no reconhecimento emocional referido por Paulmann, Pell e Kotz (2007). O conhecido envelhecimento dos sistemas auditivo (presbiacusia) e fonoarticulatório (presbifonia) de acordo com Russo (1999) e StuartHamilton (2002), colabora para que os idosos apresentem algum grau de dificuldades em processar diferentes ritmos. Na prosódia emocional, mais do que na lingüística, as variações melódicas parecem ser mais sutis e complexas, na medida em que envolvem processamento afetivo.

Enfim, o efeito de idade observado no processamento prosódico examinado nesse estudo deve-se, provavelmente, aos quadros típicos de presbiacusia e presbifonia. A artificialidade inerente aos procedimentos necessários para a avaliação da repetição e da produção de prosódia (sentenças neutras em conteúdo sem uma mensagem verbal correspondente ao sentimento utilizado) parece ter gerado uma reação de inibição, mais expressiva no processamento da prosódia emocional. Essa inibição pode ter intensificado o efeito natural da idade.

De um modo geral, a ausência de diferenças significativas entre adultos jovens e idosos nas tarefas Interpretação de atos de fala indiretos, Julgamento semântico e Prosódia lingüística repetição representa mais uma demonstração da neuroplasticidade que se estende ao longo do ciclo vital. De acordo com Dinse (2006), a reorganização cortical do cérebro do indivíduo idoso é observada tanto durante o envelhecimento propriamente dito quanto no tratamento de mudanças cognitivas relacionadas à idade.

\section{Conclusão}

No presente estudo, observou-se que o envelhecimento influenciou no processamento comunicativo de habilidades relacionadas ao papel do HD. Assim sendo, das onze provas da Bateria MAC aplicadas na amostra desta pesquisa, os adultos idosos desempenharam-se pior do que os adul-

Estud. interdiscip. envelhec., Porto Alegre, v. 12, p. 53-79, 2007. 
tos jovens em oito delas. Entretanto, de um modo geral, considerou-se que os adultos maduros foram bem-sucedidos nas habilidades comunicativas testadas. As diferenças significativas encontradas mostraram, então, um efeito de idade não indicativo de deficit lingüístico, mas sim de distintos estilos comunicativos durante a adultez ou, ainda, da existência de uma heterogeneidade no grupo de idosos não analisada nesta pesquisa.

De um modo geral, a presente pesquisa, apesar da pequena amostra e do delineamento transversal, contribuiu para que o estudo do envelhecimento das habilidades comunicativas começasse a ser mais estudado em nível nacional. $\mathrm{O}$ fato de o instrumento utilizado englobar aspectos funcionais paralingüísticos e extralingüísticos consiste em uma contribuição particular, uma vez que geralmente o desempenho de idosos é estudado com base somente em tarefas lingüísticas formais, com pouca ênfase nos processamentos prosódico e pragmático. Para que uma análise mais pormenorizada do desempenho comunicativo com o avançar da idade seja efetuada, sugere-se a promoção de investigações longitudinais, com a observação de aspectos quantitativos e qualitativos apresentados nas respostas de idosos a tarefas comunicativas. Além disso, a participação de adultos de idade intermediária e de idosos longevos em estudos comparativos é crucial para que declínios significativos no processamento da linguagem sejam situados cronologicamente.

\title{
DOES AGING INFLUENCE ON RIGHT HEMISPHERE PRAG- MATIC, LEXICAL-SEMANTIC AND PROSODIC ABILITIES?
}

\begin{abstract}
Structural linguistic abilities are more related to the left hemisphere's role, while functional linguistic abilities are linked to the right hemisphere's activity. Language in the elderly adults has been frequently investigated. In the literature, however, there are researches mainly focused on structural linguistic aspects in the aged population and the functional aspects are still less explored. This study aimed to verify if aging influences on communicative performance related to the right hemisphere. Two groups formed the sample: 1) 40 young adults and 2) 40 elderly adults. Participants should have eight or more years of formal education, without self-report
\end{abstract}


of any sensorial, neurological or psychiatric disorders. In addition, the inclusion factors were performance in the Mini-Mental State Examination superior to 23 points (elderly adults), absence of depression in the Yesavage Depression Scale. The communicative performance was assessed with the Brazilian version of the Canadian instrument Protocole Montréal d'Évaluation de la Communication (Montreal Communication Evaluation Battery) - methaphor interpretation, indirect speech acts interpretation, verbal fluency, semantic judgement and prosody tasks. The data were analyzed through a Student t Test. Elderly adults showed a significant inferior performance in the majority of the communicative tasks, except for indirect speech acts interpretation, linguistic prosody - repetition and semantic judgement. The main difficulty in the communication processing observed in the elderly group did not mean a linguistic deficit; it suggested, however, an aging effect on their communicative profile or pragmatic changes.

Keywords: Communication. Language. Right Hemisphere. Aging.

\section{REFERÊNCIAS}

ASSOCIAÇÃO BRASILEIRA DE EMPRESAS DE PESQUISA. Critério de Classificação Econômica Brasil. 2003. Disponível em: <www.abep.org/codigosguias/ ABEP_CCEB.pdf>. Acesso em: 22 maio 2007.

BEAUSOLEIL, Natacha et al. Unconstrained Oral Naming Performance in Right and Left-Hemisphere-Damaged Individuals: when education overrides the lesion. Aphasiology, New York, v.17, p.143-158, 2003.

BINDER, John. Neuroanatomy of Language Processing Studied with Functional MRI. Clinical Neuroscience, New York, v.4, p.87-94, 1997.

BOTTINI, Gabriella et al. The Role of the Right Hemisphere in the Interpretation of Figurative Aspects of Language: a positron emission tomography activation study. Brain Language, New York, v. 117, p.1241-1253, 1994.

BRANDÃO, Lenisa. Estórias Pessoais e Fictícias: estudo comparativo sobre verbosidade fora do tópico em jovens e idosos. 2002. 129 f. Dissertação (Mestrado em 
Psicologia do Desenvolvimento)-Faculdade de Psicologia, Universidade Federal do Rio Grande do Sul, Porto Alegre, 2002.

BRANDÃO, Lenisa; PARENTE, Maria Alice. Os Estudos de Linguagem do Idoso neste Último Século. Estudos Interdisciplinares Sobre o Envelhecimento, Porto Alegre, v.3, p. 37-54, 2001.

BRASIL. Ministério da Saúde. Saúde Ampla e Ações de Assistência Humanitária ao Idoso. Brasília, DF, 2004. Disponível em: < http://portalwebo2.saude.gov.br/ saude/visualizar_texto.cfm?idtxt=12465. Acesso em: 28 ago. 2004.

BRICKMAN, Adam et al. Regional White Matter and Neuropsychological Functioning Across the Adult Lifespan. Biological Psychiatry, New York, v. 60, p. 444-453, 2006.

BRYAN, Janet; LUSZCZ, Mary. Measurement of Executive Function: considerations for detecting adult age differences. Journal of Clinical and Experimental Neuropsychology, Lisse, v. 22, p. 40-55, 2000.

BURKE, Deborah; HARROLD, Rose Marie. Automatic and Effortful Semantic Processes in Old Age: experimental and naturalistic approaches. In: LIGHT, Leah; BURKE, Deborah. Language, Memory and Aging. New York: Cambridge University Press, 1988. P. 100-116.

BURKE, Deborah; MACKAY, Donald; JAMES, Lori. Theoretical Approaches to Language and Aging. In: PERFECT, Timothy; MAYLOR, Elizabeth. Models of Cognitive Aging. England: Oxford University, 2000. P. 204-237.

CALDAS, Alexandre Castro. A Herança de Franz Joseph Gall. Lisboa: McGrawHill, 1999.

CASANOVA, Jordi Peña. Congreso Nacional de Neurología. Barcelona: Sociedad Española de Neurología, 1987.

CHAMPAGNE, Maud; DESAUTELS, Marie-Claude ; JOANETTE, Yves. Accounting for the Pragmatic Deficit in RHD Individuals: a multiple case study. Brain and Language, New York, v. 87, p. 10-211, 2003. 
CHAVES, Márcia; IZQUIERDO, Ivan. Differential Diagnosis Between Dementia and Depression: a study of efficiency increment. Acta Neurologica Scandinavica, Copenhagen, v. 11, p. 412-429, 1992.

DINSE, Hubert. Cortical Reorganization in the Aging Brain. Program in Brain Research, Amsterdam, v. 157, p. 57-80, 2006.

FAGUNDES, Antônio. Descrição, Definição e Registro de Comportamento. São Paulo: Edicon, 1985.

FOLSTEIN, Marshal; FOLSTEIN, Susan; McHUGH, Paul. Mini-Mental State. Journal of Psychiatry Resources, Washington, DC, v. 12, p. 189-198, 1975.

FONSECA, Rochele et al. Bateria Montreal de Avaliação da Comunicação: bateria MAC. São Paulo: Pró-Fono, 2007a.

FONSECA, Rochele et al. Processo de Adaptação da Bateria Montreal de Avaliação da Comunicação (Bateria MAC) ao Português Brasileiro. Psicologia: reflexão e crítica, Porto Alegre, v. 20, n. 2, 2007b.

GERNSBACHER, Morton; KASCHAK, Michael. Neuroimaging Studies of Language Production and Comprehension. Annual Review of Psychology, Palo Alto, v. 54, p. 91-114, 2003.

GHISLETTA, Paolo; LINDENBERGER, Ulman. Age-Based Structural Dynamics Between Perceptual Speed and knowledge in the Berlin Aging Study: direct evidence for ability dedifferentiation in old age. Psychology and Aging, Arlington, v. 18, n. 4, p.696-713, 2003.

GUTIÉRREZ-CALVO, Manuel. Inferencias en la Comprensión del Lenguaje. In: VEGA, Manuel; VEGAS, Fernando Cuetos. Psicolinguistica del Español. Madrid: Trotta, 1999. P. 231-270.

HAMM, Verneda; HASHER, Lynn. Age and the Availability of Inferences. Psychology and Aging, Arlington, v. 7, p. 56-64, 1992. 
HARLEY, Trevor. The Psychology of Language. New York: Psychology Press, 2001.

HAUSMANN, Markus; GÜNTÜRKÜN, Onur; CORBALLIS, Michael. AgeRelated Changes in Hemispheric Asymmetry Depend on Sex. Laterality, Philadelphia, v. 8, n. 3, p. 277-290, 2003.

HUGHES, Donna; BRYAN, Janet. Adult age Differences in Strategy use During Verbal Fluency Performance. Journal of Clinical and Experimental Neuropsychology, Lisse, v. 24, n. 5, p. 642-654, 2002.

JOANETTE, Yves; GOULET, Pierre; HANNEQUIN, Didier. Right Hemisphere and Verbal Communication. New York: Springer, 1990.

JOANETTE, Yves ; SKA, Bernadette ; CÔTÉ, Hélène. Protocole MEC: protocole Montreál d'évaluation de la communication. Montréal: Ortho Édition, 2004.

KISS, Ivan; ENNIS, Tricia. Age-Related Decline in Perception of Prosodic Affect. Applied Neuropsychology, San Antonio, v. 8, n. 4, p. 251-254, 2001.

LIGHT, Leah; ALBERTSON, Shirley. Comprehension of Pragmatic Implications in Young and Older Adults. In: LIGHT, Leah; BURKE, Deborah. Language, Memory and Aging. New York: Cambridge University Press, 1988. P. 133-153.

MANSUR, Letícia Lessa et al. Teste de Nomeação de Boston: desempenho de uma população de São Paulo. Pró-Fono: revista de atualização científica, São Paulo, v. 18, n. 1, p. 13-20, 2006.

MAXIM, Jane; BRYAN, Karen. Language of the Elderly: a clinical perspective. San Diego: Singular, 1994.

MC GINNIS, Debra; ZELINSKI, Elizabeth. Understanding Unfamiliar Words in Young, Young-Old, and Old-Old Adults: inferential processing and the abstraction-deficit hypothesis. Psychology and Aging, Arlington, v. 18, n. 3, p. 497-509, 2003. 
MEIER, Manfred; THOMPSON, Gary. Methodological Issues in Clinical Studies of Right Cerebral Hemisphere Dysfunction. In: HELLIGE, Joseph. Cerebral Hemisphere Asymmetry: method, theory, and application. California: Praeger, 1983. P. 46-94.

MONETTA, Laura; OUELLET-PLAMONDON, Clairelaine; JOANETTE, Yves. Age-Related Changes in the Processing of the Metaphorical Alternative Meanings of Words. Journal of Neurolinguistics, Montréal, v. 20, n. 4, p. 277-284, 2006.

PARENTE, Maria Alice de Mattos Pimenta. Cognição e Envelhecimento. Porto Alegre: Artmed, 2006.

PARENTE, Maria Alice de Mattos Pimenta; CAPUANO, Andréa; NESPOULOUS, Jean Luc. Ativação de Modelos Mentais no Recontar de Histórias por Idosos. Psicologia: reflexão e crítica, Porto Alegre, v. 12, n. 1, p. 157-172, 1999.

PAULMANN, Silke; PELL, Marc; KOTZ, Sonja. How Aging Affects the Recognition of Emotional Speech. Brain and Language, New York, v. 104, p. 262-269, April, 2007.

RADANOVIC, Marcia; MANSUR, Letícia Lessa. Performance of a Brazilian Population Sample in the Boston Diagnostic Aphasia Examination: a pilot study. Brazilian Journal of Medical and Biological Research, Ribeirão Preto, v. 35, p. 305-317, 2002.

RODRÍGUEZ-ARANDA, Cláudia; SUNDET, Kjetil. The Frontal Hypothesis of Cognitive Aging: factor structure and age effects on four frontal tests among healthy individuals. The Journal of Genetic Psychology, Provincetown, v. 167, n. 3, p. 269-287, 2006.

RUSSO, Iêda Pacheco. Distúrbios da Audição: a presbiacusia. In: RUSSO, Ieda Pacheco. Intervenção Fonoaudiológica na Terceira Idade. Rio de Janeiro: Revinter, 1999. P. 51-82.

SALTHOUSE, Timothy. The Processing-Speed Theory of Adult Age Differences in Cognition. Psychological Review, Washington, DC, v. 103, p. 403-428, 1996. 
SASSER-COEN, Jennifer. Qualitative Changes in Creativity in the Second Half of Life: a life-span developmental perspective. Journal of Creative Behavior, Hadley, v. 27, p. 18-27, 1993.

SEARLE, John. Speech Acts. Cambridge: Cambridge University, 1969.

SKA, Bernadette; GOULET, Pierre. Trouble de dénomination lors du vieillissement normal. Persée, Montréal, v. 24, n. 96, p. 112-127, 1989.

STUART-HAMILTON, Lan. A Psicologia do Envelhecimento: uma introdução. Porto Alegre: Artes Médicas, 2002.

TAYLOR Jennifer; BURKE, Deborah. Asymmetric Aging Effects of Semantic and Phonological Processes: naming in the picture-word interference task. Psychology and Aging, Arlington, v. 17, n. 4, p. 662-676, 2002.

VERHAEGHEN, Paul. Aging and Vocabulary Scores: a meta-analysis. Psychology and Aging, Arlington, v. 18, n. 2, p. 332-339, 2003.

WINGFIELD, Arthur; LINDFIELD, Kimberly; GOODGLASS, Harold. Effects of Age and Hearing Sensitivity on the Use of Prosodic Information in Spoken Word Recognition. Journal of Speech, Language and Hearing Research, Rockville, v. 43, n. 4, p. 915-925, 2000.

WINNER, Ellen; GARDNER, Howard. The Comprehension of Metaphor in BrainDamaged Patients. Brain Language, New York, v. 100, p. 717-729, 1977.

WORLD HEALTH ORGANIZATION. Country Health Indicators. 2003. Disponível em: $<$ http://www3.who.int/whosis/country/indicators.cfm?country=bra $>$. Acesso em: 29 jan. 2006.

WRIGHT, Heather Harris; NEWHOFF, Marilyn. Age-Related Differences in Inference Revision Processing. Brain and Language, New York, v. 80, p. 226-239, 2002 . 
YESAVAGE, Jerome. The Use of Self-Rating Scales for Depression in the Elderly. In: POON, Leonard. Handbook for Clinical Memory Assessment of Older Adults. Washington, DC: American Psychological Association, 1986. P. 246-368.

Recebido em: 21/06/2007

$1^{a}$ revisão: $26 / 07 / 2007$

Aceite final: 19/02/2008 